\title{
Integral Novikov conjectures and arithmetic groups containing torsion elements
}

\author{
LIZHEN JI ${ }^{1}$
}

\begin{abstract}
In this paper, we study a generalized integral Novikov conjecture for discrete groups containing nontrivial torsion elements and prove it for not necessarily torsion-free arithmetic groups of reductive algebraic groups defined over $\mathbb{Q}$ and virtually polycyclic groups. For this purpose, we prove a general criterion that this generalized integral Novikov conjecture holds for groups $\Gamma$ having finite asymptotic dimension and satisfying suitable conditions related to actions by finite subgroups on the universal space $E_{\mathcal{F}} \Gamma$ for proper actions. For arithmetic groups $\Gamma$, we show that the Borel-Serre partial compactification $\bar{X}^{\mathrm{BS}}$ is a $\Gamma$-cofinite universal space for proper actions, which is of interest independent of the application in this paper, and satisfies these other conditions as well. For virtually polycyclic groups $\Gamma$, we use the filtration induced from the canonical decreasing commuting series to understand the structure of fixed-point sets on a model $E_{\mathcal{F}} \Gamma$ given by homogeneous spaces.
\end{abstract}

\section{Introduction}

The original Novikov conjecture is concerned with oriented homotopy invariance of higher signatures of manifolds (see [27] for a survey and references). It is known that this conjecture is equivalent to the rational injectivity of the assembly map

$$
A: H_{*}(B \Gamma, \mathbb{L}(\mathbb{Z})) \rightarrow L_{*}(\mathbb{Z} \Gamma),
$$

where $\Gamma$ is the fundamental group of the manifolds, and $L_{*}(\Gamma \mathbb{Z})$ are the surgery groups; the rational injectivity of $A$ means that $A \otimes \mathbb{Q}: H_{*}(B \Gamma$, $\mathbb{L}(\mathbb{Z})) \otimes \mathbb{Q} \rightarrow L_{*}(\mathbb{Z} \Gamma) \otimes \mathbb{Q}$ is injective. If the assembly map $A$ is split injective, then the left side $H_{*}(B \Gamma, \mathbb{L}(\mathbb{Z}))$ can be used to compute a part of the important but difficult right side $L_{*}(\mathbb{Z} \Gamma)$. The conjecture that the integral assembly map $A$ is injective is called the integral Novikov conjecture in

\footnotetext{
${ }^{1}$ Partially supported by NSF grant DMS 0405884.
} 
L-theory. There are also assembly maps in other theories, for example, in the algebraic $K$-theory,

$$
A: H_{*}(B \Gamma, \mathbb{K}(\mathbb{Z})) \rightarrow K_{*}(\mathbb{Z} \Gamma),
$$

where $K_{*}(\mathbb{Z} \Gamma)$ are the algebraic groups of the (group) ring $\mathbb{Z} \Gamma$. The rational injectivity of this map $A$, i.e., the injectivity of $A \otimes \mathbb{Q}$, in Equation (1.2) is called the Novikov conjecture in (algebraic) $K$-theory, and the injectivity of $A$ is called the integral Novikov conjecture in (algebraic) $K$-theory.

If $\Gamma$ contains nontrivial torsion elements, then it is expected that in general the assembly map $A$ in both cases fails to be injective, and hence the integral Novikov conjecture does not hold. On the other hand, many natural and important groups such as $\mathrm{SL}(n, \mathbb{Z})$ are not torsion free (see $[1$, Section 2]), and it is important to modify the assembly map in order to obtain an integral Novikov conjecture for such groups.

Assume that $\Gamma$ contains torsion elements. Let $\mathcal{F}$ be the family of all finite subgroups of $\Gamma$. Let $E_{\mathcal{F}} \Gamma$ be the universal space for the family $\mathcal{F}$, i.e., for proper actions of $\Gamma$. (Note that the space $E_{\mathcal{F}} \Gamma$ is also denoted by $\underline{E} \Gamma$.) Briefly, it is characterized as follows: (1) $E_{\mathcal{F}} \Gamma$ is contractible. (2) The stabilizer in $\Gamma$ of any point of $E_{\mathcal{F}} \Gamma$ is a finite subgroup of $\Gamma$. (3) For any finite subgroup $H \subset \Gamma$, the fixed-point set $\left(E_{\mathcal{F}} \Gamma\right)^{H}$ is nonempty and weakly contractible.

Let $p t$. be the trivial $\Gamma$-space consisting of one point. Then $H_{*}^{\Gamma}(p t$., $\mathbb{L}(\mathbb{Z})))=L_{*}(\mathbb{Z} \Gamma)$ and $\left.H_{*}^{\Gamma}(p t ., \mathbb{K}(\mathbb{Z}))\right)=K_{*}(\mathbb{Z} \Gamma)$, and the constant map from $E_{\mathcal{F}} \Gamma$ to $p t$. induces assembly maps:

$$
\left.\left.A: H_{*}^{\Gamma}\left(E_{\mathcal{F}} \Gamma, \mathbb{L}(\mathbb{Z})\right)\right) \rightarrow L_{*}(\mathbb{Z} \Gamma), \quad A: H_{*}^{\Gamma}\left(E_{\mathcal{F}} \Gamma, \mathbb{K}(\mathbb{Z})\right)\right) \rightarrow K_{*}(\mathbb{Z} \Gamma) .
$$

The injectivity of the assembly map $A$ in Equation (1.3) is called the generalized integral Novikov conjecture in $L$-theory and $K$-theory, respectively. See $[28,29,30,31,32]$ for related results and assembly maps for groups containing torsion elements. In general, for groups containing torsion elements, the integral assembly maps in Equation (1.3) are not surjective (see [23]). To get isomorphisms, we need to enlarge the family $\mathcal{F}$ to the family of virtually cyclic subgroups (see $[25,24,26]$ ). For convenience, we also call this the integral Novikov conjecture for groups containing torsion elements. When $\Gamma$ is torsion free, $E_{\mathcal{F}} \Gamma$ is equal to $E \Gamma$, which is the universal space for proper and fixed-point free actions and hence equal to the universal covering space of $B \Gamma$; in this case,

$$
H_{*}^{\Gamma}\left(E_{\mathcal{F}} \Gamma, \mathbb{L}(\mathbb{Z})\right)=H_{*}(B \Gamma, \mathbb{L}(\mathbb{Z})),
$$


and hence the generalized integral Novikov conjecture specializes to the previous integral Novikov conjecture for torsion-free groups in Equations (1.1) and (1.2).

The integral Novikov conjecture was proved in [2] for all torsion-free arithmetic subgroups of all linear algebraic groups $\mathbf{G}$ over $\mathbb{Q}$ whether $\mathbf{G}$ is reductive or not. In this paper, we are mainly concerned with groups which contain nontrivial torsion elements.

It was proved in [1] that this generalized integral Novikov conjecture in both $K$ - and $L$-theories holds for all $S$-arithmetic subgroups of reductive algebraic groups $\mathbf{G}$ of rank 0 over a global field, i.e., either a number field or a function field. The purpose of this paper is to remove the rank 0 assumption and prove the conjecture for all arithmetic subgroups of reductive algebraic groups over number fields. (See [3] for a summary of Novikov conjectures for discrete subgroups of Lie groups and related groups, in particular, arithmetic subgroups and $S$-arithmetic subgroups of algebraic groups.)

In [1], the rank 0 assumption is important in order to apply the following result of Rosenthal $[4,5,22]$.

Theorem 1.1. Assume that $\Gamma$ admits a cofinite $\Gamma-\mathrm{CW}$-complex $E_{\mathcal{F}} \Gamma$ which has a metrizable compactification $\overline{E_{\mathcal{F}} \Gamma}$ satisfying the following conditions:

1. The action of $\Gamma$ on $E_{\mathcal{F}} \Gamma$ extends to a continuous action on $\overline{E_{\mathcal{F}} \Gamma}$.

2. For any finite subgroup $H$, the set of fixed points $\left(\overline{E_{\mathcal{F}} \Gamma}\right)^{H}$ is contractible and contains $E_{\mathcal{F}} \Gamma^{H}$ as a dense subset. In particular, when $H$ is trivial, $\overline{E_{\mathcal{F}} \Gamma}$ is contractible.

3. The action of $\Gamma$ is small at the infinity of $\overline{E_{\mathcal{F}} \Gamma}$.

Then the generalized integral Novikov conjecture in both $K$ - and L-theory holds for $\Gamma$.

In fact, the rank 0 assumption of $\mathbf{G}$ is needed to give a cofinite $\Gamma$-CWcomplex $E_{\mathcal{F}} \Gamma$ and a small compactification $\overline{E_{\mathcal{F}} \Gamma}$. If the rank of $\mathbf{G}$ is positive, it seems difficult to construct such a compactification and apply this result. But many algebraic groups such as $\mathrm{SL}(n)$ have positive rank.

To overcome this difficulty, we combine the results in $[4,5,6]$ and prove the following result. When $\Gamma$ is torsion free, it is reduced to the result that asdim $\Gamma<+\infty$ and the existence of a finite $B \Gamma$ imply the integral Novikov conjectures for $\Gamma$, proved in $[6,7,8,35,36]$.

Theorem 1.2. Assume that $\Gamma$ has finite asymptotic dimension and admits a cofinite $\Gamma-\mathrm{CW}$-complex $E_{\mathcal{F}} \Gamma$. For any pair of finite subgroups $H, I$ of $\Gamma$, 
$I \subseteq H$, let $N_{H}(I)$ be the normalizer of $I$ in $H$. Assume that for any such a pair $H$ and $I$, the set of fixed points $\left(E_{\mathcal{F}} \Gamma\right)^{I}$ and the quotient $N_{H}(I) \backslash\left(E_{\mathcal{F}} \Gamma\right)^{I}$ are uniformly contractible and of bounded geometry. Then the generalized integral Novikov conjecture in both $K$ - and L-theories holds for $\Gamma$.

We recall from [9] that a metric space $(X, d)$ is called uniformly contractible if there is a positive function $f(R)$ with $f(R) \geq R$ for $R>0$, such that for any $x \in X$, the ball $B(x, R)$ can be contracted to a point in the larger ball $B(x, f(R))$. It is said to be of bounded geometry (or coarsely bounded) if there exist a positive constant $\varepsilon$ and a positive function $g(R)$ such that the number of elements of any $\varepsilon$-separated subset in any ball $B(x, R)$ is bounded by $g(R)$.

The first condition in Theorem 1.2 follows from the following result of [2, Section 3].

Proposition 1.3. Any arithmetic subgroup $\Gamma$ of a linear algebraic group $\mathbf{G}$ defined over $\mathbb{Q}$ has finite asymptotic dimension.

In this paper, we will show that if $\mathbf{G}$ is reductive, then the other conditions in Theorem 1.2 are also satisfied by $\Gamma$. Briefly, let $X$ be the symmetric space associated with the real locus $G=\mathbf{G}(\mathbb{R})$. Then the Borel-Serre partial compactification $\bar{X}^{\mathrm{BS}}$ in [10] is a $\Gamma$-cofinite $E_{\mathcal{F}} \Gamma$ (Theorem 3.2). This explicit model can be used to show that other conditions on the fixed-point sets in Theorem 1.2 are also satisfied.

As a corollary, we obtain the result mentioned earlier (see also Theorem 3.1 below).

Corollary 1.4. Let $\mathbf{G}$ be a reductive algebraic group defined over $\mathbb{Q}$. Then for every arithmetic subgroup $\Gamma \subset \mathbf{G}(\mathbb{Q})$, which is not necessarily torsion free, the generalized integral Novikov conjecture in both $K$ - and L-theories holds for $\Gamma$.

Another corollary (Theorem 4.1) shows that the generalized integral Novikov conjecture holds for all virtually polycyclic groups (see [4] and Remark 4.2 below). It is conceivable that this result allows one to remove the assumption that $\mathbf{G}$ is reductive in the above corollary by using the Levi decomposition of nonreductive algebraic groups.

A natural question is whether the same result holds for $S$-arithmetic subgroups of semisimiple algebraic groups over number fields. In this case, $E_{\mathcal{F}} \Gamma$ can be taken as the product of a symmetric space with Bruhat-Tits buildings. But one difficulty is to check the conditions on the fixed-point 
sets and their quotients in Theorem 1.2. On the other hand, the rational injectivity of the assembly map in both $K$ - and $L$-theories holds for these $S$-arithmetic subgroups by [11] (see [12, Proposition 3.3] and [34]); and when the symmetric space associated with $\mathbf{G}$ is the real hyperbolic space, then the generalized integral Novikov conjectures hold for every $S$-arithmetic subgroup of $\mathbf{G}(k)$ [12, Theorem 3.2].

The rest of this paper is organized as follows. In Section 2, we prove Theorem 1.2 by combining methods in $[4,5,6]$. In Section 3 , we first show that $\bar{X}^{\mathrm{BS}}$ is a cofinite $\Gamma$-CW-complex $E_{\mathcal{F}} \Gamma$ for arithmetic subgroups $\Gamma$. Then we show that finite quotients of the sets of fixed points in $\bar{X}^{\text {BS }}$ are uniformly contractible and of bounded geometry, and hence the generalized integral Novikov conjecture holds for arithmetic subgroups. In Section 4, we prove the generalized integral Novikov conjecture for virtually polycyclic groups by using the canonical filtration of an associated simply connected solvable group by the descending commutator series to describe the fixed-point sets of finite subgroups on a suitable model of $E_{\mathcal{F}} \Gamma$.

Remark 1.5. The first version of this paper was finished in March 2006. After sending this paper to David Rosenthal on June 6, 2006, I received from him the paper [13] by Bartels and Rosenthal, which contains results [13, Theorems A and B and corollaries] similar to but stronger than Theorem 1.2 in this paper. It should be stressed that this paper and [13] were carried out completely independently of each other. It is also helpful to point out that the result in Theorem 3.2 has been used in literature (see [14, Remark 5.5] and [33]), but the proof here is the first one written up. Similar results in Remark 3.5 on the existence of a cofinite $E_{\mathcal{F}} \Gamma$-space for $S$-arithmetic groups are also of independent interest.

\section{The generalized integral Novikov conjecture}

We first recall the notion of coarse structures and models in terms of controlled algebras for both sides of the assembly map. For simplicity, we only consider the case of algebraic $K$-theory. So we will construct small catagories of modules associated with the coarse structures whose images under the nonconnective $K$-theory functor, $\mathbb{K}^{-\infty}$, denoted by $\mathbb{K}$ for simplicity in the following, give the desired spectra. We will mostly follow the notations in [6].

Definition 2.1. Let $X$ be a topological space. A coarse structure on $X$ is a collection $\mathbb{E}$ of subsets of $X \times X$ satisfying the following conditions: 
1. $\mathbb{E}$ is closed under finite union.

2. $\mathbb{E}$ is closed under composition.

3. For any $E \in \mathbb{E}$, its inverse $E^{\mathrm{op}}=\{(y, x) \mid(x, y) \in E\}$ is contained in some element of $\mathbb{E}$.

4. For any $E \in \mathbb{E}$ and any compact subset $K \subset X$, the set $\{x \mid(k, x)$ or $(x, k) \in E$ for some $k \in K\}$ has a compact closure.

Definition 2.2. Let $X$ be a topological space, and $\mathbb{E}$ be a coarse structure on $X$. Let $\mathcal{A}$ be a small additive category.

1. A geometric $\mathcal{A}$-module $M$ with support contained in $\mathbb{E}$ consists of a collection of objects $M_{x} \in \mathcal{A}$, where $x \in X$, such that the support, $\operatorname{supp}(M)=\left\{x \mid M_{x} \neq 0\right\}$, is locally finite and the image of $\operatorname{supp}(M)$ under the diagonal embedding into $X \times X$ is contained in some element of $\mathbb{E}$.

2. A morphism between two geometric $\mathcal{A}$-modules $M, N$ with support contained in $\mathbb{E}$ is a collection of morphisms $\phi_{x, y}: M_{y} \rightarrow N_{x}$ such that for each fixed $x$ (resp. $y$ ), $\phi_{x, y} \neq 0$ for only a finite number of $y$ (resp. $x$ ), and

$$
\operatorname{supp}(\phi)=\left\{(x, y) \in X \times X \mid \phi_{x, y} \neq 0\right\}
$$

is contained in some element in $\mathbb{E}$.

3. The category $\mathcal{A}(\mathbb{E})$ has objects given by geometric $\mathcal{A}$-modules supported in $\mathbb{E}$ and morphisms supported in $\mathbb{E}$.

An important example of small additive categories $\mathcal{A}$ is the category of finitely generated free $\mathbb{Z}$-modules.

Given a collection $\mathcal{S}$ of subsets of $X$ closed under finite union, there is a coarse structure $\mathbb{E}_{\mathcal{S}}$, called the restriction of $\mathbb{E}$ to $\mathcal{S}$, defined by

$$
\begin{aligned}
\mathbb{E}_{\mathcal{S}}= & \left\{E \subset X \times X \mid E \subset E^{\prime} \text { for some } E^{\prime} \in \mathbb{E}, \text { and } \pi_{1}(E),\right. \\
& \left.\pi_{2}(E) \subset F \text { for some } F \in \mathcal{S}\right\},
\end{aligned}
$$

where $\pi_{1}, \pi_{2}$ are the projections on the two factors. There is also an enlargement $\overline{\mathcal{S}}$ of $\mathcal{S}$ by $\mathbb{E}$, defined by

$$
\overline{\mathcal{S}}=\left\{\pi_{1}(E \circ \operatorname{Diag}(F)) \mid E \in \mathbb{E}, F \in \mathcal{S}\right\}
$$


For any such $\mathcal{S}$, there are two important categories: $\mathcal{A}\left(\mathbb{E}_{\overline{\mathcal{S}}}\right)$, the category of geometric $\mathcal{A}$-modules with support contained in $E_{\overline{\mathcal{S}}}$, and the Karoubi quotient of $\mathcal{A}(\mathbb{E})$ by $\mathcal{A}\left(\mathbb{E}_{\overline{\mathcal{S}}}\right)$, denoted by $\mathcal{A}(\mathbb{E}) / \mathcal{S}$.

The coarse structures that we will use in this paper are the continuous control for a topological space and the bounded control for a metric space.

\section{Definition 2.3.}

1. Let $X$ be a proper metric space. The collection of all bounded subsets $E$ of $X \times X$ gives the bounded coarse structure, denoted by $\mathbb{B}(X)$.

2. Let $X$ be a topological space. A subset $E$ of $(X \times[0,1))^{2}$ is said to be continuously controlled at $(x, 1)$ if for any neighborhood $U$ of $(x, 1)$ in $X \times[0,1]$, there is another neighborhood $V$ of $(x, 1)$ such that

$$
(X \times[0,1]-U) \times V \cap E=\emptyset, \quad V \times(X \times[0,1]-U) \cap E=\emptyset .
$$

The collection of all subsets of $(X \times[0,1))^{2}$ that are continuously controlled at all points $(x, 1)$ and for any compact subset $K \subset X \times[0,1)$, $E \cap \pi_{1}^{-1}(K)$ and $E \cap \pi_{2}^{-1}(K)$ both have compact closures forms the continuously controlled coarse structure on $X \times[0,1)$ and is denoted by $\mathbb{J}(X)$.

3. Let $X$ be a metric space. The coarse structure $\mathbb{J}_{b}(X)$ of continuous control with bounded control in $X$-direction consists of all $E \in$ $\mathbb{J}(X)$ such that the subset $\{x \mid(x, t) \in E$ for some $t \in[0,1)\} \subset X$ is bounded.

Since $X \times\{1\}$ is contained in the boundary of $X \times[0,1)$, the continuous control is at the boundary.

For any topological space $X$, let $\mathcal{S}$ consist of only $X \times\{0\}$ and define

$$
\mathcal{A}(\mathbb{J}(X))_{\infty}=\mathcal{A}(\mathbb{J}(X)) / \mathcal{S}
$$

If $X$ is furthermore a metric space, define

$$
\mathcal{A}\left(\mathbb{J}_{b}(X)\right)_{\infty}=\mathcal{A}\left(\mathbb{J}_{b}(X)\right) / \mathcal{S}
$$

It is known by $\left[6\right.$, Lemma 3.5] that $\mathcal{A}\left(\mathbb{J}_{b}(X)\right)_{\infty}=\mathcal{A}(\mathbb{J}(X))_{\infty}$.

Let $X=E_{\mathcal{F}} \Gamma$ be endowed with a $\Gamma$-invariant length structure. Let $\mathcal{A}$ be the category of finitely generated free $\mathbb{Z}$-modules. Then $\Gamma$ acts on 
$\mathcal{A}\left(\mathbb{J}\left(E_{\mathcal{F}} \Gamma\right)\right)_{\infty}$. It is known (see [6, Theorem 3.12]) that

$$
\begin{aligned}
H_{*}^{\Gamma}\left(E_{\mathcal{F}} \Gamma, \mathbb{K}(\mathbb{Z})\right)= & \pi_{*}\left(\mathbb{K}\left(\mathcal{A}\left(\mathbb{J}\left(E_{\mathcal{F}} \Gamma\right)_{\infty}\right)\right)^{\Gamma}\right)=\pi_{*}\left(\mathbb{K}\left(\mathcal{A}\left(\mathbb{J}_{b}\left(E_{\mathcal{F}} \Gamma\right)_{\infty}\right)\right)^{\Gamma}\right), \\
& K_{*}(\mathbb{Z} \Gamma)=\pi_{*}\left(\mathbb{K} \mathcal{A}\left(\mathbb{B}\left(E_{\mathcal{F}} \Gamma\right)\right)^{\Gamma}\right) .
\end{aligned}
$$

It is also known [6, Remark 3.8 and Equation (5.2)] that there is an assembly map

$$
A: K_{n} \mathcal{A}\left(\mathbb{J}_{b}\left(E_{\mathcal{F}} \Gamma\right)_{\infty}\right) \rightarrow K_{n-1} \mathcal{A}\left(\mathbb{B}\left(E_{\mathcal{F}} \Gamma\right)\right)
$$

induced from the Karoubi sequence

$$
\mathcal{A}\left(\mathbb{B}\left(E_{\mathcal{F}} \Gamma\right)\right) \rightarrow \mathcal{A}\left(\mathbb { J } _ { b } ( E _ { \mathcal { F } } \Gamma ) \rightarrow \mathcal { A } \left(\mathbb{J}_{b}\left(E_{\mathcal{F}} \Gamma\right)_{\infty} .\right.\right.
$$

By the same proof as in [4, Proposition 6.2], we can prove

Proposition 2.4. With the above notation, $\mathbb{K}(\mathbb{Z} \Gamma) \cong \mathbb{K} \mathcal{A}\left(\mathbb{B}\left(E_{\mathcal{F}} \Gamma\right)\right)^{\Gamma}$ and hence

$$
K_{*}(\mathbb{Z} \Gamma)=\pi_{*}\left(\mathbb{K} \mathcal{A}\left(\mathbb{B}\left(E_{\mathcal{F}} \Gamma\right)\right)^{\Gamma}\right) .
$$

The induced map on the sets of $\Gamma$-fixed points from Equation (2.1) gives the assembly map in the generalized integral Novikov conjecture in Equation (1.3):

$$
A: H_{*}^{\Gamma}\left(E_{\mathcal{F}} \Gamma, \mathbb{K}(\mathbb{Z})\right) \rightarrow K_{*}(\mathbb{Z} \Gamma)
$$

As in $[4,6]$, let

$$
S=\mathbb{K} \mathcal{A}\left(\mathbb{J}_{b}\left(E_{\mathcal{F}} \Gamma\right)_{\infty}\right), \quad T=\mathbb{K} \mathcal{A}\left(\mathbb{B}\left(E_{\mathcal{F}} \Gamma\right)\right) .
$$

Since $S, T$ are $\Gamma$-equivariant spectra, we have homotopy fixed sets $S^{h \mathcal{F} \Gamma}, T^{h \mathcal{F} \Gamma}$ with respect to the family $\mathcal{F}$ of finite subgroups of $\Gamma$ :

$$
S^{h \mathcal{F} \Gamma}=\operatorname{Map}_{\Gamma}\left(E_{\mathcal{F}} \Gamma, S\right), \quad T^{h \mathcal{F} \Gamma}=\operatorname{Map}_{\Gamma}\left(E_{\mathcal{F}} \Gamma, T\right) .
$$

When $\Gamma$ is torsion free, they are the usual homotopy fixed sets

$$
S^{h \Gamma}=\operatorname{Map}_{\Gamma}(E \Gamma, S), \quad T^{h \Gamma}=\operatorname{Map}_{\Gamma}(E \Gamma, T) .
$$

Since $S^{\Gamma}=\operatorname{Map}_{\Gamma}(p t ., S), T^{\Gamma}=\operatorname{Map}_{\Gamma}(p t ., T)$, the map from $E_{\mathcal{F}} \Gamma$ to a point $p t$. induces two maps

$$
S^{\Gamma} \rightarrow S^{h \mathcal{F} \Gamma}
$$




$$
T^{\Gamma} \rightarrow T^{h \mathcal{F} \Gamma} .
$$

The assembly map in Equation (2.1) also induces a map on the homotopy fixed sets:

$$
S^{h \mathcal{F} \Gamma} \rightarrow T^{h \mathcal{F} \Gamma} .
$$

If the maps $S^{\Gamma} \rightarrow S^{h \mathcal{F} \Gamma}$ and $S^{h \mathcal{F} \Gamma} \rightarrow T^{h \mathcal{F} \Gamma}$ are isomorphism, then the assembly map in Equation (2.2), $A: \pi_{*}\left(S^{\Gamma}\right) \rightarrow \pi_{*}\left(T^{\Gamma}\right)$, is a split injection, and hence the generalized integral Novikov conjecture is proved.

In [6, Theorem 6.1, Proposition 5.5], the following result is proved.

Proposition 2.5. If $E_{\mathcal{F}} \Gamma$ is a proper geodesic space of finite asymptotic dimension, uniformly contractible and of bounded geometry, then the assembly map $A: S \rightarrow T$ in Equation (2.1) is an isomorphism.

Corollary 2.6. If $\Gamma$ has finite asymptotic dimension and $E_{\mathcal{F}} \Gamma$ is a cofinite $\Gamma$-CW-complex, then $A: S \rightarrow T$ is an isomorphism.

Proof. Under the assumption, $E_{\mathcal{F}} \Gamma$ is a geodesic space (or admits a length structure) induced from a metric on the quotient $\Gamma \backslash E_{\mathcal{F}} \Gamma$. Since $\Gamma \backslash E_{\mathcal{F}} \Gamma$ is a finite CW-complex, $E_{\mathcal{F}} \Gamma$ is uniformly contractible and of bounded geometry. Since $\Gamma$ is coarsely equivalent to $E_{\mathcal{F}} \Gamma$ and has finite asymptotic dimension, $E_{\mathcal{F}} \Gamma$ has also finite asymptotic dimension. Hence all the conditions in the above proposition are satisfied, and the assembly map $A: S \rightarrow T$ is an isomorphism.

By the same proof as in [4, Theorem 6.3 and Corollary 6.4], we can prove

Proposition 2.7. With the above notation, the map in Equation (2.3) is an isomorphism $S^{\Gamma} \cong S^{h \mathcal{F} \Gamma}$.

The most difficult step is to show that the map in Equation (2.5) is an isomorphism:

$$
S^{h \mathcal{F} \Gamma} \cong T^{h \mathcal{F} \Gamma} .
$$

The proof is similar to the proof of [4, Theorem 7.1]. We outline the proof in [4, Theorem 7.1] and mention the modifications needed. The basic ingredient is the following result [4, Lemma 4.1].

Proposition 2.8. Let $A: S \rightarrow T$ be an equivariant map between two spectra with $\Gamma$-action. Assume that $E_{\mathcal{F}} \Gamma$ is a cofinite $\Gamma-\mathrm{CW}$-complex. If for 
every finite group $G \in \mathcal{F}$, the induced map on the fixed sets $A^{G}: S^{G} \rightarrow T^{G}$ is a weak homotopy equivalence, then $S^{h \mathcal{F} \Gamma} \cong T^{h \mathcal{F} \Gamma}$.

In [4, Theorem 7.1], the proof goes as follows. For each finite subgroup $G$, there is a $G$-equivariant filtration of $X=E_{\mathcal{F}} \Gamma$ by $G$-conjugacy classes of subsets of fixed points of subgroups contained in $G$. Note that for the trivial group, the fixed set is $X$, and for $G$, it is $X^{G}$, the smallest subspace in this filtration.

This induces filtrations of $S^{G}$ and $T^{G}$, and the isomorphism between $S^{G}$ and $T^{G}$ is proved by induction on the strata of these filtrations. In the proof, the contractibility of $X^{H}$ (or rather unions of suitable $G$-conjugacy classes of $\left.X^{H}\right), H \subset G$, together with the fact that the quotient of $X^{H}$ by $N_{G}(H)$, the normalizer of $H$ in $G$, has the trivial reduced Steenrod homology is crucial, due to [4, Proposition 5.4].

In our case, these conditions are replaced by

1. $X^{H}$ is uniformly contractible and of bounded geometry,

2. each quotient $N_{G}(H) \backslash X^{H}$ is also uniformly contractible and of bounded geometry.

Then Corollary 2.6 above replaces [4, Proposition 5.4] and the proof of [4, Theorem 7.1] goes through and Theorem 1.2 is proved.

\section{Arithmetic groups}

In this section, we apply Theorem 1.2 to prove the following result.

Theorem 3.1. Let $\mathbf{G}$ be a reductive algebraic group defined over $\mathbb{Q}, \Gamma \subset$ $\mathbf{G}(\mathbb{Q})$ any arithmetic subgroup. Then the generalized integral Novikov conjecture in both $K$ - and L-theories holds for $\Gamma$.

As mentioned earlier, it was proved in [2] that for every torsion-free arithmetic subgroup of any linear algebraic group defined over $\mathbb{Q}$ which is not necessarily reductive, the integral Novikov conjecture holds. Hence, the new case occurs when $\Gamma$ contains nontrivial torsion elements.

To prove this theorem, we start with the construction of $E_{\mathcal{F}} \Gamma$. Let $G=$ $\mathbf{G}(\mathbb{R})$ be the real locus of $\mathbf{G}, K \subset G$ a maximal compact subgroup. Then $X=G / K$ with a $G$-invariant Riemannian metric is a symmetric space of nonpositive curvature (i.e., no compact factor). Since $X$ is simply connected and nonpositively curved, it is diffeomorphic to an Euclidean space. It is known that $\Gamma$ acts properly on $X$. Using the fact that $X$ is nonpositively 
curved, it is easy to see that for any finite subgroup $H$ of $\Gamma$, its set of fixed points $X^{H}$ is a nonempty totally geodesic submanifold, in particular simply connected and nonpositively curved (see [1, Lemma 4.13]), and hence contractible. This implies that $X$ is a $E_{\mathcal{F}} \Gamma$ space.

It is known that $\Gamma \backslash X$ is compact if and only if the $\mathbb{Q}$-rank of $\mathbf{G}$ is zero. When $\Gamma \backslash X$ is compact, the existence of $\Gamma$-equivariant triangulation of $X$ (see [15] or other references in [1]) shows that $X$ is a cofinite $\Gamma$-CW-complex $E_{\mathcal{F}} \Gamma$. If $\Gamma \backslash X$ is noncompact, we need the Borel-Serre compactification to construct a cofinite $\Gamma$-CW-complex $E_{\mathcal{F}} \Gamma$.

Assume that the $\mathbb{Q}$-rank of $\mathbf{G}$ is positive. Let $\bar{X}^{\mathrm{BS}}$ be the Borel-Serre partial compactification of $X$ [10] (see also [16]), which is obtained by adding at infinity the boundary faces $e(\mathbf{P})$ of $\mathbb{Q}$-parabolic subgroups $\mathbf{P}$ :

$$
\bar{X}^{\mathrm{BS}}=X \cup \coprod_{\mathbf{P}} e(\mathbf{P}),
$$

where $\mathbf{P}$ runs over all (proper) $\mathbb{Q}$-parabolic subgroups of $\mathbf{G}$. It should be emphasized that $\bar{X}^{\mathrm{BS}}$ is not compact and hence not a compactification. It is often called the Borel-Serre bodification. More specifically, for each (proper) parabolic subgroup $\mathbf{P}$ of $\mathbf{G}$, its boundary component $e(\mathbf{P})$ is defined as follows: Let $P=\mathbf{P}(\mathbb{R})$ be the real locus of $\mathbf{P}$, and $N_{P}$ the real locus of the unipotent radical $\mathbf{N}_{\mathbf{P}}$ of $\mathbf{P}$. For each basepoint $x_{0} \in X$ or a corresponding maximal compact subgroup $K$ of $G$, there is a $\mathbb{Q}$-Langlands decomposition of $P$ :

$$
P=N_{P} A_{\mathbf{P}, x_{0}} M_{\mathbf{P}, x_{0}} \cong N_{P} \times A_{\mathbf{P}, x_{0}} \times M_{\mathbf{P}, x_{0}},
$$

where $A_{\mathbf{P}, x_{0}}$ is the lift of a $\mathbb{Q}$-split component $A_{\mathbf{P}}$ of $\mathbf{P} / \mathbf{N}_{\mathbf{P}}(\mathbb{R})$ into $P$, which is stable under the Cartan involution associated with $K$. Similarly, $M_{\mathbf{P}}=\mathbf{M}_{\mathbf{P}}(\mathbb{R})$, and $M_{\mathbf{P}, x_{0}}$ is a lift into $P$, which is also stable under the Cartan involution associated with $K$. (Note that $\mathbf{P} / \mathbf{N}_{\mathbf{P}}$ is an algebraic group defined over $\mathbb{Q}$.) It is known that

$$
P \cap K=M_{\mathbf{P}, x_{0}} \cap K
$$

and it is a maximal compact subgroup of the reductive group $M_{\mathbf{P}, x_{0}}$, which is often denoted by $K_{\mathbf{P}, x_{0}}$. Define the boundary symmetric space $X_{\mathbf{P}}$ associated with $\mathbf{P}$ by

$$
X_{\mathbf{P}}=M_{\mathbf{P}, x_{0}} / K_{\mathbf{P}, x_{0}}
$$


The subscript $x_{0}$ is often dropped if the reference to the base-point $x_{0}$ is clear. Then the boundary component $e(\mathbf{P})$ is defined by

$$
e(\mathbf{P})=N_{P} \times X_{\mathbf{P}}
$$

The Langlands decomposition of $P$ induces the horospherical decomposition of $X$ :

$$
X \cong N_{P} \times A_{\mathbf{P}} \times X_{\mathbf{P}}
$$

A sequence of points in $X$ converges to a boundary point in $e(\mathbf{P})$ if and only if the components in $N_{P}$ and $X_{\mathbf{P}}$ converge to those components of the boundary point, and the $A_{\mathbf{P}}$-component goes to infinity through the positive chamber associated with $\mathbf{P}$ and the distance to the chamber walls goes to infinity (see [16]).

\section{Example.}

A simple but important example to keep in mind is the case when $\mathbf{G}=\mathrm{SL}(2)$, and $X=\mathrm{SL}(2, \mathbb{R}) / \mathrm{SO}(2) \cong \mathbb{H}$, the upper half plane. Clearly $X$ has a natural boundary $\mathbb{R} \cup\{\infty\}$. There is an one-to-one correspondence between the rational boundary points in $\mathbb{Q} \cup\{\infty\} \subset \mathbb{R} \cup\{\infty\}$ and proper $\mathbb{Q}$-parabolic subgroups of $\mathrm{SL}(2)$.

It is known that $\bar{X}^{\mathrm{BS}}$ is a real analytic manifold with corners, and the $\Gamma$-action on $X$ extends to a proper real analytic action on $\bar{X}^{\mathrm{BS}}$. Under this action, for any $\gamma \in \Gamma$, a boundary component $e(\mathbf{P})$ is mapped to $e\left({ }^{\gamma} \mathbf{P}\right)$. If $\Gamma$ is torsion free, then the quotient $\Gamma \backslash \bar{X}^{\mathrm{BS}}$ is a compact real analytic manifold with corners. Consequently, the inclusion $\Gamma \backslash \bar{X} \hookrightarrow \Gamma \backslash \bar{X}^{\mathrm{BS}}$ is a homotopy equivalence.

In the above example of $X=\mathrm{SL}(2, \mathbb{R}) / \mathrm{SO}(2)=\mathbb{H}, \Gamma \backslash \bar{X}^{\mathrm{BS}}$ is a surface with boundary, which is obtained by adding a circle to every end (or cusp) of $\Gamma \backslash X$.

Theorem 3.2. For an arithmetic subgroup $\Gamma$ as above, the Borel-Serre partial compactification $\bar{X}^{\mathrm{BS}}$ is a cofinite $\Gamma$-CW-complex $E_{\mathcal{F}} \Gamma$.

Proof. The existence of $\Gamma$-equivariant triangulation [15] shows that $\bar{X}^{\mathrm{BS}}$ has the structure of a cofinite $\Gamma-\mathrm{CW}$-complex. We need to show that for any finite subgroup $H$ of $\Gamma$, the set of fixed points $\left(\bar{X}^{\mathrm{BS}}\right)^{H}$ is contractible.

If $H$ is trivial, then $\left(\bar{X}^{\mathrm{BS}}\right)^{H}=\bar{X}^{\mathrm{BS}}$. Since $\bar{X}^{\mathrm{BS}}$ is a manifold with corners whose interior is equal to $X$ and $X$ is contractible, it is clear that $\bar{X}^{\mathrm{BS}}$ is contractible. 
Assume that $H$ is a nontrivial finite subgroup. Since $X$ is simply connected and nonpositively curved, $X^{H}$ is a nonempty totally geodesic submanifold (see [1, the proof of Proposition 4.5]). If $H$ does not fix any boundary point, then $\left(\bar{X}^{\mathrm{BS}}\right)^{H}=X^{H}$ and is hence contractible. Suppose that $H$ does fix a boundary point $z \in e(\mathbf{P})$ for some $\mathbb{Q}$-parabolic subgroup $\mathbf{P}$. It follows from the construction of $\bar{X}^{\mathrm{BS}}$ that for any element $\gamma \in \Gamma, \gamma$ maps a boundary component $e(\mathbf{P})$ to $e\left({ }^{\gamma} \mathbf{P}\right)$. Since $\mathbf{P}$ is equal to its normalizer, it follows that $H \subset P$. Pick any point $x_{0} \in X^{H}$. Then $H$ fixes $x_{0}$ and hence $H$ is contained in the maximal subgroup $K_{x_{0}}$ of $G$ that fixes $x_{0}$. By Equation (3.1), $H \subset P \cap K=K_{\mathbf{P}, x_{0}} \subset M_{\mathbf{P}, x_{0}}$. This implies that $H \subset \Gamma \cap M_{\mathbf{P}, x_{0}}$.

The above discussion implies that for any $\mathbb{Q}$-parabolic subgroup $\mathbf{P}, H$ fixes a point in $e(\mathbf{P})$ if and only if $H \subset M_{P, x_{0}}$ for any point $x_{0} \in X^{H}$. Let $Z_{N_{P}} H$ be the subspace of elements of $N_{P}$ that commute with $H$, and $\left(X_{\mathbf{P}}\right)^{H}$ be the set of fixed points of $H$ in $X_{\mathbf{P}}$. It can be shown that $Z_{N_{P}} H \times\left(X_{\mathbf{P}}\right)^{H}$ is the set $e(\mathbf{P})^{H}$ of fixed points in $e(\mathbf{P})$.

Since $A_{P, x_{0}}$ commutes with $M_{\mathbf{P}, x_{0}}$, it can be shown that the fixed-point set $X^{H}$ contains the following subset:

$$
Z_{N_{P}} H \times A_{\mathbf{P}, x_{0}} \times\left(X_{\mathbf{P}}\right)^{H} .
$$

This implies that the boundary fixed points $e(\mathbf{P})^{H}$ can be contracted into $X^{H}$ along the direction of $A_{\mathbf{P}, x_{0}}$.

Since

$$
\left(\bar{X}^{\mathrm{BS}}\right)^{H}=X^{H} \cup \coprod_{\mathbf{P}} e(\mathbf{P})^{H},
$$

where $\mathbf{P}$ runs over all $\mathbb{Q}$-parabolic subgroups containing $H$, it follows that the boundary points of $\left(\bar{X}^{\mathrm{BS}}\right)^{H}$ can be contracted into the interior $X^{H}$, which is contractible. Therefore, $\left(\bar{X}^{\mathrm{BS}}\right)^{H}$ is contractible.

Remark 3.3. The retraction of the boundary points of $\bar{X}^{\mathrm{BS}}$ into $X$ and hence the boundary points of $\left(\bar{X}^{\mathrm{BS}}\right)^{H}$ into $X^{H}$ can be written down explicitly. See [17] for details. It can also be shown that $\left(\bar{X}^{\mathrm{BS}}\right)^{H}$ is a manifold with corners.

Remark 3.4. This theorem was mentioned in [14, Remark 5.8], attributed to Borel and Prasad, but without any indication of their proof. Since it is difficult to find the original correspondences between Adem, Borel and Prasad, we give a proof here. 
Remark 3.5. The result in Theorem 3.2 can be generalized to $S$-arithmetic subgroups over number fields such as $\operatorname{SL}\left(n, \mathbb{Z}\left[\frac{1}{p_{1}}, \ldots, \frac{1}{p_{m}}\right]\right)$. Briefly, let $\Gamma$ be an $S$-arithmetic subgroup of a reductive algebraic group $\mathbf{G}$ defined over a number field $k$. For every finite place $\mathfrak{p}$ in $S$, i.e., $\mathfrak{p} \in S_{f}$, there is a BruhatTits building $X_{\mathfrak{p}}$ associated with $\mathbf{G}\left(k_{\mathfrak{p}}\right)$. To the set $S_{\infty}$ of all the infinite places, there is associated a symmetric space $X_{\infty}$. Define

$$
X_{S}=X_{\infty} \times \prod_{\mathfrak{p} \in S_{f}} X_{\mathfrak{p}}
$$

Since every of $X_{\infty}$ and $X_{\mathfrak{p}}$ is a proper CAT(0)-space, it follows that $X_{S}$ is also a proper CAT(0)-space (see $[1,12]$ for more details about $X_{\mathfrak{p}}$ and $X_{S}$ ). This implies that $X_{S}$ is a $E_{\mathcal{F}} \Gamma$-space. If the $k$-rank of $\mathbf{G}$ is equal to 0 , then $\Gamma \backslash X_{S}$ is compact. By [1, Proposition 2.13], $X_{S}$ is a cofinite $\Gamma$-CW-complex. On the other hand, if the $k$-rank of $\mathbf{G}$ is positive, then there is a Borel-Serre partial compactification

$$
\bar{X}_{S}^{\mathrm{BS}}=\bar{X}_{\infty}^{\mathrm{BS}} \times \prod_{\mathfrak{p} \in S_{f}} X_{\mathfrak{p}}
$$

such that the quotient $\Gamma \backslash \bar{X}_{S}^{\mathrm{BS}}$ is compact. By the same arguments as in the proof of Theorem 3.2, we can show that $\bar{X}_{S}^{\mathrm{BS}}$ is a $E_{\mathcal{F}} \Gamma$-space. Arguments similar to those in the proof of [1, Proposition 2.13] show that $\bar{X}_{S}^{\mathrm{BS}}$ has the structure of a cofinite $\Gamma$-CW-complex. Therefore, $\bar{X}_{S}^{\mathrm{BS}}$ is a model of $E_{\mathcal{F}}$ given by a cofinite $\Gamma$-CW-complex.

Next we show that (1) $E_{\mathcal{F}} \Gamma=\bar{X}^{\mathrm{BS}}$ is metrizable, (2) with respect to a suitable metric, it is a proper geodesic space, uniformly contractible and of bounded geometry, (3) and the fixed-point sets of finite subgroups of $\Gamma$ also satisfy the conditions in Theorem 1.2.

Since $\bar{X}^{\mathrm{BS}}$ is a manifold with corners, it is clearly metrizable. On the other hand, $X$ is a complete Riemannian manifold with respect to the $G$ invariant metric, and hence the boundary points in $e(\mathbf{P})$ are of infinite distance from the interior points in $X$ with respect to the invariant metric. This implies that the induced distance function on $X$ does not extend to a distance function on $\bar{X}^{\mathrm{BS}}$.

For this purpose, we need a realization of $\bar{X}^{\text {BS }}$ as a submanifold $X_{T}$ of $X$, where $T$ is a suitable truncation parameter (see [17]). Briefly, $X_{T}$ is submanifold with corners of $X$ obtained by removing a cusp neighborhood of every boundary component $e(\mathbf{P})$ in a $\Gamma$-equivariant way with respect to the 
fixed basepoint $x_{0}$ corresponding to $K \subset G$. When $X$ is the upper halfplane $\mathbb{H}, X_{T}$ is obtained from $\mathbb{H}$ by removing a horoball at every rational boundary point in $\mathbb{Q} \cup\{\infty\} \subset \mathbb{R} \cup\{\infty\}$. [The sizes of these balls are determined by the equivariance with respect to the action of $\operatorname{SL}(2, \mathbb{Q})$.]

The group $\Gamma$ acts properly on $X_{T}$ with a compact quotient $\Gamma \backslash X_{T}$, which is diffeomorphic to the Borel-Serre compactification $\Gamma \backslash \bar{X}^{\mathrm{BS}}$. The space $\Gamma \backslash X_{T}$ can also be obtained from $\Gamma \backslash X$ by truncating every end. Since $X_{T}$ is a subspace of $X$, the Riemannian length function of $X$ restricts to a distance function $d_{T}$ on $X_{T}$. But $X_{T}$ is not a convex subspace of $X$, and hence the restriction $d_{T}$ to $X_{T}$ is not a length function. On the other hand, the distance function $d_{T}$ induces a length function $\tilde{d}_{T}$, with respect to which $X_{T}$ is a proper geodesic space.

To apply Theorem 1.2, we need to show that for any pair of finite subgroups $H, I$ of $\Gamma$ with $I \subseteq H$, the fixed-point set $\left(X_{T}\right)^{I}$ and the quotient $N_{H}(I) \backslash\left(X_{T}\right)^{I}$ are uniformly contractible and of bounded geometry.

Since the quotient $\Gamma \backslash X_{T}$ is a compact manifold with corners, by the proof of Corollary 2.6, $\left(X_{T}, \tilde{d}_{T}\right)$ is of bounded geometry.

For any finite subgroup $I \subset \Gamma$, as mentioned earlier, $X^{I}$ is a totally geodesic submanifold of $X$ (see [1, the proof of Proposition 4.5]), and hence $\left(X_{T}\right)^{I}=X^{I} \cap X_{T}$ is of bounded geometry.

To show the desired uniform contractibility of $\left(X_{T}\right)^{I}$ and $N_{H}(I) \backslash\left(X_{T}\right)^{I}$, we need to compare the subspace distance $d_{T}$ and the induced geodesic distance function $\tilde{d}_{T}$ on $X_{T}$. From the construction, it is clear that $\tilde{d}_{T} \geq d_{T}$. In the other direction, we have the following result.

Proposition 3.6. There exists a positive function $f(t)$ satisfying $\lim _{t \rightarrow+\infty}$ $f(t)=+\infty$ such that for any $x, y \in X_{T}$,

$$
\tilde{d}_{T}(x, y) \leq f\left(d_{T}(x, y)\right)
$$

In other words, $d_{T}$ and $\tilde{d}_{T}$ are coarsely equivalent.

Proof. Since $\Gamma \backslash X_{T}$ is compact and both $d_{T}$ and $\tilde{d}_{T}$ are $\Gamma$-invariant distance functions on $X_{T}$, it suffices to set $x$ equal to a fixed base-point $x_{0}$. For any $R>0$, consider the closed metric ball $B_{X_{T}}\left(x_{0}, R\right)$ with respect to the metric $d_{T}$. Let $f(R)$ be the maximum distance from $x_{0}$ to points in $B_{X_{T}}\left(x_{0}, R\right)$ with respect to $\tilde{d}_{T}$. Then for any $y \in B_{X_{T}}\left(x_{0}, R\right)$,

$$
\tilde{d}_{T}(x, y) \leq f(R)
$$


Now for any point $y \in X_{T}$, take $R=d_{T}\left(x_{0}, y\right)$. The above inequality implies that

$$
\tilde{d}_{T}\left(x_{0}, y\right) \leq f\left(d_{T}\left(x_{0}, y\right)\right) .
$$

Remark 3.7. A stronger conclusion that $f$ is a linear function holds when $\Gamma$ is an irreducible lattice and the rank of the symmetric space $X$ is at least 2. In this case, there exists a constant $C \geq 1$ such that

$$
d_{T} \leq \tilde{d}_{T} \leq C d_{T} .
$$

This is a restatement of the main theorem of [18]. In fact, since $\Gamma \backslash X_{T}$ is compact, $\left(X_{T}, \tilde{d}_{T}\right)$ is quasi-isometric to $\Gamma$ with a word length $d_{W}$. It is proved in [18] that $d_{W}$ is Lipschitz equivalent to the induced metric on an orbit $\Gamma x_{0}$ in $X$ from $d$, and the upper bound in the equation follows from this.

Proposition 3.8. The proper geodesic spaces $\left(X_{T}, \tilde{d}_{T}\right)$ and $\left(X_{T}\right)^{I}$, where $I \subset \Gamma$ is any finite subgroup, are uniformly contractible.

Proof. For any $x \in X$, let $B_{X}(x, R)$ be the metric ball in $X$ with center $x$ and radius $R$ in $X$. For any $x \in X_{T}$, let $B_{X_{T}}(x, R)$ be the metric ball in $X_{T}$ with respect to $d_{T}$, and $\tilde{B}_{X_{T}}(x, R)$ be the metric ball in $X_{T}$ with respect to the length function $\tilde{d}_{T}$. By Proposition 3.6, $\tilde{B}_{X_{T}}(x, R)$ is contained in $B_{X_{T}}(x, f(R))$. We claim that for any $r>0$, the ball $B_{X_{T}}(x, r)$ is contractible. In fact, the ball $B_{X}(x, r)$ in $X$ is clearly contractible. Now $B_{X_{T}}(x, r)$ is obtained from $B_{X}(x, r)$ by removing disjoint and contractible pieces of horoballs. In fact, $B_{X_{T}}(x, r)=B_{X}(x, r) \cap X_{T}$, and $X_{T}$ is obtained from $X$ by removing a disjoint $\Gamma$-equivariant family of horoballs, and $X$ can be deformation retracted to $X_{T}$, equivariantly with respect to any arithmetic subgroup $\Gamma$, along the geodesics induced from the geodesic action of the split component $A_{\mathbf{P}}$ associated with $\mathbf{P}$ (see [17]). (Note that $X_{T}$ is a manifold with corners with one boundary face corresponding to every $\mathbb{Q}$-parabolic subgroup of $\mathbf{G}$, and the geodesic flow of $A_{\mathbf{P}}$ is orthogonal to the boundary face corresponding to $\mathbf{P}$.) This implies that $B_{X}(x, r)$ can be deformation retracted in $X$ to $B_{X_{T}}(x, r)$ by following along parts of these geodesics, and hence $B_{X_{T}}(x, r)$ is contractible. This proves that $\left(X_{T}, \tilde{d}_{T}\right)$ is uniformly contractible.

To prove the second statement, we note that for any finite subgroup $I \subset \Gamma$, the fixed-point set $X^{I}$ is a totally geodesic submanifold, and $X_{T}^{I}$ is equal to $X_{T} \cap X^{I}$ and is also a totally geodesic submanifold of $\left(X_{T}, \tilde{d}_{T}\right)$. 
Then the uniform contractibility of $X_{T}^{I}$ follows from that of $X_{I}$ as in the above paragraph.

Proposition 3.9. For any pair of finite subgroups $I, J$ of $\Gamma$ such that $J$ stabilizes the fixed-point set $X_{T}^{I}$, the quotient $J \backslash X_{T}^{I}$ is uniformly contractible.

Proof. Since $X_{T}^{I}$ is a totally geodesic submanifold of $X_{T}$, without loss of generality, we assume $X_{T}^{I}=X_{T}$ and show that $J \backslash X_{T}$ is uniformly contractible.

As in the previous proposition, we first show that $J \backslash X$ is uniformly contractible. By [1], the fixed-point set $X^{J}$ is a nonempty totally geodesic submanifold. If $X^{J}=X$, then $J$ acts trivially on $X$ and $J \backslash X^{J}=X$. Hence $J \backslash X^{J}$ is uniformly contractible in this case. Assume that $X^{J}$ is a proper submanifold. Let $N\left(X^{J}\right)$ be the normal bundle in $X$. Clearly $J$ acts on $N\left(X^{J}\right)$. Since $X$ is nonpositively curved and simply connected, the exponential map

$$
\exp : N\left(X^{J}\right) \rightarrow X
$$

is a diffeomorphism. Since $J$ acts isometrically on $X$, the map exp is $J$ equivariant. Therefore, we have a homeomorphism (or a diffeomorphism between orbifolds):

$$
\exp : J \backslash N\left(X^{J}\right) \rightarrow J \backslash X
$$

Let $x_{0} \in X^{J}$ and $V$ be the normal space $N_{x_{0}}\left(X^{J}\right)$. Then $J$ acts on $V$ by linear transformation with the origin as the only fixed point, and the quotient $J \backslash V$ is a cone. In fact, if $J$ has any other fixed point in $V$, it will contradict the assumption that $V$ is normal to $X^{J}$. This implies that the quotient $J \backslash X$ a cone bundle on $X^{J}$ with the fiber cone given by $J \backslash V$. We endow $J \backslash V$ with the metric induced from the restriction of the invariant metric to $N_{x_{0}}\left(X^{J}\right)$. Since the sectional curvature of $X$ is nonpositive, the map exp in Equation (3.3) is distance increasing.

First, we note that the cone $J \backslash V$ is uniformly contractible. Let 0 denote the origin of $V$ and also the vertex of the cone $J \backslash V$. We consider several cases. First, assume that $J$ acts freely on $V-\{0\}$. Then there exists a positive function $f(R)$ such that for any $x \in J \backslash V$ with $d(x, 0) \geq f(R)$, the ball $B(x, R)$ in $J \backslash V$ is diffeomorphic to a ball of the same radius in $V$ and hence contractible. Certainly we can take $f$ so that $f(R) \geq R$. On the other hand, if the distance $d(0, x) \leq f(R)$, then the ball $B(x, f(R))$ contains 0 and 
hence $B(x, f(R))$ is contractible to 0 along the rays from 0 in $J \backslash V$. This implies that the ball $B(x, R)$ is either contractible already or contractible to the point 0 in the larger ball $B(x, f(R))$. This proves that $J \backslash V$ is uniformly contractible.

Second, we assume that some elements of $J$ fix some points in $V-\{0\}$. Clearly, for any $\gamma \in J$, the set of fixed points $V^{\gamma}$ is a linear subspace, and near the image of $V^{\gamma}, J \backslash V$ has a cone structure. As in the above paragraph, we can consider balls that are close to these fixed points and balls that are far away to show that $J \backslash V$ is uniformly contractible.

Using the fact that the exp in Equation (3.3) is distance preserving on the radial direction and expanding in other directions, similar arguments as above show that the quotient $J \backslash X$ is uniformly contractible.

As in the proof of Proposition 3.8, we can use the uniform contractibility of $J \backslash X$ and the $\Gamma$-equivariance of the deformation retract of $X$ to $X_{T}$ in [17] to show that $J \backslash X_{T}$ is also uniformly contractible. This completes the proof of Proposition 3.9.

Proof of Theorem 3.1. Let $\Gamma$ be an arithmetic group as in the theorem. By [2, Section 3] (see Proposition 1.3 above), $\Gamma$ has a finite asymptotic dimension. By Theorem 3.2 and Proposition 3.9, the conditions in Theorem 1.2 are satisfied by $\Gamma$. Therefore, the generalized integral Novikov conjecture holds for $\Gamma$.

\section{Polycyclic groups}

In this section, we show that the generalized integral Novikov conjecture holds for polycyclic groups.

Recall that a group $\Gamma$ is called polycyclic if there exists a finite sequence of subgroups

$$
\Gamma=\Gamma_{0} \supset \Gamma_{1} \supset \cdots \supset \Gamma_{k}=\{e\}
$$

such that $\Gamma_{i}$ is normal in $\Gamma_{i-1}$ and $\Gamma_{i-1} / \Gamma_{i}$ is cyclic. If each quotient $\Gamma_{i-1} / \Gamma_{i}$ is isomorphic to $\mathbb{Z}, \Gamma$ is called strongly polycyclic. A group $\Gamma$ is called virtually polycyclic (resp. virtually strongly polycyclic) if it contains a ploycyclic (resp. strongly ploycyclic) group $\Gamma^{\prime}$ as a subgroup of finite index.

It is known [19, Theorem 4.28] that any lattice (which is automatically cocompact) in a simply connected solvable Lie group is strongly polycyclic, and every polycyclic group $\Gamma$ admits a normal subgroup $\Gamma^{\prime}$ of finite index that is isomorphic to a lattice in a solvable simply connected Lie group. 
More generally, let $S$ be a connected, simply connected solvable group and $K$ a compact subgroup of the automorphism group $\operatorname{Aut}(S)$. Let $S \rtimes K$ be the associated semidirect product. It is known [37, Theorem 3] that any discrete cocompact subgroup $\Gamma$ of $S \rtimes K$ is virtually polycyclic, and $\Gamma$ does not contain any nontrivial finite normal subgroup; and the converse is also true.

In this section, we prove the following result.

Theorem 4.1. If $\Gamma$ is a virtually polycyclic group, then the generalized integral Novikov conjecture in both $L$ - and $K$-theories holds for $\Gamma$.

Remark 4.2. This result was proved in [4, Section 9 and p. 141]. But the proof there seems to be problematic. Realize $\Gamma$ or a quotient of $\Gamma$ by a maximal finite normal subgroup as a lattice in the group $S \rtimes K$. Then $S$ can be taken to be a $E_{\mathcal{F}} \Gamma$ space. It was stated in [4, Section 9 and p. 141] that $S$ has the structure of a cofinite $\Gamma$-CW-complex, but no proof was given. (It is not entirely obvious. See Proposition 4.4 below.) The paper then claimed that since $S$ is diffeomorphic to an Euclidean space, $E_{\mathcal{F}} \Gamma=S$ can be compactified by adding a sphere at infinity as in the case of an Euclidean space $\mathbb{R}^{n}$. Actually, unless $\Gamma$ is crystallographic, this compactification is not $\Gamma$-equivariant. In fact, it is not clear how to extend the action of $\Gamma$ on $S$ to the compactification since the boundary points do not have intrinsic interpretations as in the case of the Euclidean space in terms of geodesics. On the other hand, in a special case when $S$ endowed with the invariant metric is nonpositively curved, it is contained in [1, Proposition 4.5], which states that if a group $\Gamma$ acts properly and co-compactly on a complete, nonpositively curved manifold $M$, then the generalized integral Novikov conjecture in both $K$ - and $L$-theories holds for $\Gamma$. (In this case, $M$ can be compactified by adding the set $M(\infty)$ of equivariance classes of geodesics, and the isometric action of $\Gamma$ on $M$ clearly extends to a countinuous action on $M \cup M(\infty)$.) This special case happens, for example, if there is a semisimple Lie group $G$ with an Iwasawa decomposition $G=N A K$, and $S=N A$.

To prove Theorem 4.1, the idea is to apply Theorem 1.2. The first condition is satisfied according to the following result.

Proposition 4.3. If $\Gamma$ is virtually polycyclic, and $\Gamma^{\prime}$ a polycyclic subgroup of finite index, then the asymptotic dimension of $\Gamma$, denoted by asdim $\Gamma$, is bounded by the Hirsch length of $\Gamma^{\prime}$ and hence is finite. 
Proof. The proposition follows from [20, Theorems 1 and 8]. Since finite groups and cyclic groups have finite asymptotic dimension, the finiteness of asdim $\Gamma$ also follows directly from [21, Theorem 3].

The other conditions concern the existence of a $E_{\mathcal{F}} \Gamma$ space given by a cofinite $\Gamma$-CW-complex.

Proposition 4.4. If $\Gamma$ is a virtually polycyclic group, then it admits a $E_{\mathcal{F}} \Gamma$-space given by a cofinite $\Gamma$-CW-complex.

Proof. By definition, there exists a polycyclic subgroup $\Gamma^{\prime}$ of finite index. It is known that any polycyclic group contains a torsion-free subgroup of finite index. In fact, it contains a strongly polycyclic subgroup of finite index which is torsion free (see [19, Lemma 4.6]). Hence, $\Gamma$ contains a torsion-free subgroup of finite index. This implies that there is a uniform bound on the order of finite subgroups of $\Gamma$, and hence there are maximal normal finite subgroups contained in $\Gamma$. Let $H$ be such a maximal subgroup. Then $H \backslash \Gamma$ is a virtually polycyclic group that does not contain a nontrivial finite normal group. By [37, Theorem 3] mentioned earlier, there exists a connected and simply connected solvable group $S$ and a compact subgroup $K \subset \operatorname{Aut}(S)$ such that $H \backslash \Gamma$ is isomorphic to a cocompact discrete subgroup of $S \rtimes K$. Since $K$ is a maximal compact subgroup of $S \rtimes K$, it is known (see [33, Theorem 4.4] for example) that $S=S \rtimes K / K$ is diffeomorphic to an Euclidean space and gives a $E_{\mathcal{F}} \Gamma$-space.

Since $S$ is a smooth manifold and $\Gamma$ acts smoothly and properly, the existence of equivariant triangulation in [15] shows that $S$ is a cofinite $\Gamma$ $\mathrm{CW}$-complex, and hence gives a $\Gamma$-cofinite $E_{\mathcal{F}} \Gamma$-space.

To apply Theorem 1.2, we need to understand fixed-point sets $S^{H}$ of finite subgroups $H$ of $\Gamma$ and show that their quotients by finite groups are uniformly contractible and of bounded geometry. Our discussion below also gives a more direct proof of the above proposition that $S$ is a $E_{\mathcal{F}} \Gamma$-space.

Let $S^{1}=[S, S], S^{i+1}=\left[S^{i}, S^{i}\right]$, for $i \geq 1$. Since $S$ is solvable, we get a finite descending commutator series

$$
S \cong S^{0} \supseteq S^{1} \supseteq \cdots \supseteq S^{k-1} \supseteq S^{k}=\{e\}
$$

for some $k \geq 0$. Since all $S^{i}$ are connected and simply connected, each successive quotient $S^{i-1} / S^{i}$ is isomorphic as Lie groups to $\mathbb{R}^{d_{i}}$ for some $d_{i} \geq 1$.

A simple but crucial observation is that the filtration in Equation (4.1) is canonical in the sense that they are invariant under $\operatorname{Aut}(S)$, and hence 
$\operatorname{Aut}(S)$ acts on each successive quotient $S^{i-1} / S^{i}=\mathbb{R}^{d_{i}}$ as an affine transformation.

Since $S^{1}$ is a normal subgroup of $S=S^{0}, S$ is a principal $S^{1}$-bundle over the base $S^{0} / S^{1}$. Denote the projection map by $\pi_{1}: S^{0} \rightarrow S^{0} / S^{1}$. Since $S^{0} / S^{1} \cong \mathbb{R}^{d_{1}}$, this bundle can be trivialized and hence there is a diffeomorphism

$$
S \cong S^{0} / S^{1} \times S^{1}
$$

For any $s \in S$, the left multiplication on $S$ induces a translation on $S^{0} / S^{1} \cong \mathbb{R}^{d_{1}}$. For any $v_{1} \in S^{0} / S^{1}$ fixed by $s$, the multiplication by $s$ induces a left multiplication on $\pi^{-1}(v) \cong S^{1}$ by some element in $S^{1}$.

Similarly, $S^{1}$ is a principal $S^{2}$-bundle over $S^{1} / S^{2}=\mathbb{R}^{d_{2}}$, and hence $S^{1}$ is diffeomorphic to $S^{1} / S^{2} \times S^{2}$. By induction, $S$ has the structure of a multi-step fibration with the successive bases (from the base up) given by $S / S^{1}, S^{1} / S^{2}, \ldots, S^{k-1} / S^{k}$. It is important to note that this multi-step fibration is invariant under $\operatorname{Aut}(S)$. Similarly, by induction, we get a diffeomorphism

$$
S \cong S / S^{1} \times S^{1} / S^{2} \times \cdots \times S^{k-1} / S^{k} \cong \mathbb{R}^{d_{1}} \times \cdots \times \mathbb{R}^{d_{k}}
$$

The left multiplication of $S$ on $S$ induces an action on $S / S^{1} \times S^{1} / S^{2} \times$ $\cdots \times S^{k-1} / S^{k}$. For any $i \leq k$, it induces an action on $S / S^{1} \times S^{1} / S^{2} \times \cdots \times$ $S^{i-1} / S^{i}$, which is a part of the multi-step fibration of $S$.

For any $s \in S \rtimes K$ and $v=\left(v_{1}, \ldots, v_{k}\right) \in S$ according to the decomposition in Equation (4.3), if the action of $s$ on $S / S^{1} \times S^{1} / S^{2} \times \cdots \times S^{i-1} / S^{i}$ fixes $\left(v_{1}, \ldots, v_{i}\right)$, then it induces an affine map on $S^{i} / S^{i+1}$.

Proposition 4.5. Let $S$ be a connected and simply connected solvable Lie group, $K \subset \operatorname{Aut}(S)$ a compact subgroup, and $H \subset S \rtimes K$ a finite subgroup as above. Then the set $S^{H}$ of fixed points by $H$ on $S=S \rtimes K / K$ has a structure of multi-step fibration contained in the multi-step fibration of $S$ discussed above (Equation 4.3) such that the subset contained in each base $S^{i-1} / S^{i}$ is an affine subspace $V_{i} \subset \mathbb{R}^{d_{i}}$, and hence $S^{H}$ is diffeomorphic to

$$
V_{1} \times \cdots \times V_{k} \subset \mathbb{R}^{d_{1}} \times \cdots \times \mathbb{R}^{d_{k}} \cong S / S^{1} \times \cdots \times S^{k-1} / S^{k}
$$

under the decomposition in Equation (4.3). In particular, the set $S^{H}$ is contractible.

Proof. By the discussions above, both $K$ and $S$ induce affine actions on $S / S^{1}$. If $H$ fixes a point $v=\left(v_{1}, \ldots, v_{k}\right)$ according to the decomposition in 
Equation (4.3), then it must fix the first component $v_{1} \in S / S^{1} \cong \mathbb{R}^{d_{1}}$; and hence $v_{1}$ belongs to the set $V_{1}$ of fixed points of a finite affine group on $S / S^{1} \cong \mathbb{R}^{d_{1}}$. Clearly $V_{1}$ is an affine space.

For each $v_{1} \in V_{1}, H$ induces a finite group action on $\pi_{1}^{-1}\left(v_{1}\right) \cong S^{1}$, where $\pi_{1}: S \rightarrow S / S^{1}$. In fact, for any $\gamma \in H$ and $v_{1}=g S^{1} \in S / S^{1}$, assume that $\gamma g S^{1}=g S^{1}$. Then $g^{-1} \gamma g S^{1}=S^{1}$ and hence $g^{-1} \gamma g \in S^{1} \rtimes \operatorname{Aut}\left(S^{1}\right)$. This implies that $H \subset g\left(S^{1} \rtimes \operatorname{Aut}\left(S^{1}\right) \cap S \rtimes \operatorname{Aut}(S)\right) g^{-1}$. By the same argument as above, its set of fixed points by $H$ in $\pi^{-1}\left(v_{1}\right) \cong S^{1} / S^{2}$ is an affine subspace $V_{2}$. For different points in $v_{1} \in V_{1}$, the sets of fixed points in $\pi^{-1}\left(v_{1}\right)$ are isomorphic affine subspaces.

Hence, the set of fixed points of $H$ in $S / S^{2}$ is a fibration with base in $S / S^{1}$ given by an affine space $V_{1}$ and fibers in $S^{1} / S^{2}$ given by isomorphic affine spaces $V_{2}$. By induction, we can prove that $S^{H}$ has the structure of a multi-step fibration in the proposition.

Proposition 4.6. Let $H$ be a finite subgroup of $S \rtimes K$ as in the above proposition. Let $I$ be a finite subgroup of $S \rtimes K$ stabilizing the set $S^{H}$. Then the quotient $I \backslash S^{H}$ is uniformly contractible and of bounded geometry.

Proof. In the multi-step fibration of $S$ in Equation (4.3) above, each base $S^{i-1} / S^{i}$ has a canonical Euclidean metric induced from the invariant Riemannian metric on $S$ and the induced action of a subgroup of $S \rtimes K$ on $S=S \rtimes K / K$ is given by isometry. By the previous proposition, it suffices to show that finite quotients of an Euclidean space is a uniformly contractible. Since such quotients are metric cones over some lower-dimensional Euclidean spaces, by the same (in fact, slightly simpler) proof of Proposition 3.9, we can show that such quotients are uniformly contractible, i.e., by considering metric balls either far away from the cone vertex point or sufficiently close to the convex vertex, we can show that they are either contractible in themselves or in slightly larger balls. Since such metric cones over Euclidean spaces are of bounded geometry, it can be proved that $I \backslash S^{H}$ is of bounded geometry.

Proof of Theorem 4.1. It follows from Propositions 4.4 and 4.6 that the conditions in Theorem 1.2 are satisfied for virtually polycyclic groups. Hence, Theorem 4.1 is proved.

\section{References}

[1] L. Ji, The integral Novikov conjectures for S-arithmetic groups I, K-theory, 2007. 
[2] L. Ji, Asymptotic dimension and the integral K-theoretic Novikov conjecture for arithmetic groups, J. Diff. Geom. 68 (2004), 535-544.

[3] L. Ji, Large scale geometry, compactifications and the integral Novikov conjectures for arithmetic groups, Proceedings of the Third International Congress of Chinese Mathematicians, AMS-IP, 2007, in press.

[4] D. Rosenthal, Splitting with continuous control in algebraic K-theory, K-Theory 32 (2004), 139-166.

[5] D. Rosenthal, Continuous control and the algebraic L-theory assembly map, Forum Math. 18 (2006), 193-209.

[6] A. Bartel, Squeezing and higher algebraic K-theory, K-Theory 28 (2003), 19-37.

[7] S. Chang, S. Ferry and G. Yu, Bounded rigidity of manifolds and asymptotic dimension growth, K-theory, 2007.

[8] A. Dranishnikov, S. Ferry and S. Weinberger, An etale approach to the Novikov conjecture, Preprint, math.GT/0509644.

[9] N. Higson and J. Roe, On the coarse Baum-Connes conjecture, in 'Novikov conjectures, index theorems and rigidity', vol. 2, Cambridge University Press, 1995, 227-254.

[10] A. Borel and J.P. Serre, Corners and arithmetic Groups, Comment. Math. Helv. 48 (1973), 436-491.

[11] M. Bökstedt, W. Hsiang and I. Madsen, The cyclotomic trace and algebraic K-theory of spaces, Invent. Math. 111 (1993), 465-539.

[12] L. Ji, The integral Novikov conjectures for S-arithmetic groups II, unpublished manuscript, 2006.

[13] A. Bartels and D. Rosenthal, On the K-theory of groups with finite asymptotic dimension, Preprint 2006, arXiv:math.KT/0605088.

[14] A. Adem, and Y. Ruan, Twisted orbifold K-theory, Comm. Math. Phys. 237 (2003), 533-556.

[15] S. Illman, Existence and uniqueness of equivariant triangulations of smooth proper $G$-manifolds with some applications to equivariant Whitehead torsion, J. Reine Angew. Math. 524 (2000), 129-183.

[16] A. Borel and L. Ji, Compactifications of symmetric and locally symmetric spaces, Birkhäuser, 2006, 479+ix. 
[17] L. Saper, Tilings and finite energy retractions of locally symmetric spaces, Comment. Math. Helv. 72 (1997), 167-202.

[18] A. Lubotzky, S. Mozes and M. Raghunathan, The word and Riemannian metrics on lattices of semisimple groups, Inst. Hautes Études Sci. Publ. Math. 91 (2000), 5-53.

[19] M. Raghunathan, Discrete subgroups of Lie groups, Springer-Verlag, 1972.

[20] G. Bell and A. Dranishnikov, A Hurewicz-type theorem for asymptotic dimension and applications to geometric group theory, Trans. Amer. Math. Soc. 358 (2006), 4749-4764.

[21] G. Bell and A. Dranishnikov, On asymptotic dimension of groups, Algebr. Geom. Topol. 1 (2001), 57-71.

[22] G. Carlsson and E. Pedersen, Controlled algebra and the Novikov conjectures for $K$ - and L-theory, Topology 34 (1995), 731-758.

[23] T. Farrell, A remark on $K_{0}$ of crystallographic groups, Topol. Appl. 26 (1987), 97-99.

[24] F. Farrell and L. Jones, The lower algebraic $K$-theory of virtually infinite cyclic groups, K-Theory 9 (1995), 13-30.

[25] F. Farrell and L. Jones, Isomorphism conjectures in algebraic K-theory, JAMS 6 (1993), 249-297.

[26] F. Farrell and L. Jones, The surgery L-groups of poly-(finite or cyclic) groups, Invent. Math. 91 (1988), 559-586.

[27] S. Ferry, A. Ranicki and J. Rosenberg, Novikov conjectures, index theorems and rigidity, vol. 1 and 2; Cambridge University Press, 1995.

[28] F. Quinn, Algebraic K-theory of poly-(finite or cyclic) groups, Bull. Amer. Math. Soc. 12 (1985), 221-226.

[29] S. Weinberger, The topological classification of stratified spaces, University of Chicago Press, Chicago, 1994, xiv+283 pp.

[30] M. Yamasaki, L-groups of virtually polycyclic groups, Topol. Appl. 33 (1989), 223-233. 
[31] M. Yamasaki, Lattices of a Lie group and Seifert fibrations, Michigan Math. J. 35 (1988), 215-225.

[32] M. Yamasaki, L-groups of crystallographic groups, Invent. Math. 88 (1987), 571-602.

[33] W. Lück, Survey on classifying spaces for families of subgroups, in Infinite groups: geometric, combinatorial and dynamical aspects, pp. 269322, Progr. Math., 248, Birkhäuser, 2005.

[34] E. Guentner, N. Higson and S. Weinberger, The Novikov conjecture for linear groups, Publ. Math. Inst. Hautes Études Sci. No. 101 (2005) 243-268.

[35] G. Yu, The Novikov conjecture for groups with finite asymptotic dimension, Ann. of Math. 147 (1998) 325-355.

[36] G. Carlsson and B. Goldfarb, The integral K-theoretic Novikov conjecture for groups with finite asymptotic dimension, Invent. Math. 157 (2004) 405-418.

[37] B.Wilking, Rigidity of group actions on solvable Lie groups, Math. Ann. 317 (2000) 195-237.

Center of Mathematical Sciences

ZHEJIANG UNIVERSITY

HANGZHOU 310027

CHINA

Department of Mathematics

UNIVERSITY OF MICHIGAN

AnN Arbor, MI 48109

USA

E-mail address: 1ji@umich.edu

RECEIVEd July 7, 2006 